\title{
Multi-omics Revealed the Global Responses of Bovine Alveolar Macrophages to Mycobacterium tuberculosis /Mycobacterium bovis
}

Rong Wei ( 952257684@qq.com )

the People's Hospital of Suzhou New District

Yuqi Chen

the People's Hospital of Suzhou New District

Huiya Ma

Northwest A\&F University

Yangbo Duan

Northwest A\&F University

Xueyan Ma

Northwest A\&F University

Lihui Tan

the People's Hospital of Suzhou New District

Jianjian Dong

the People's Hospital of Suzhou New District

Chenkai Jin

the People's Hospital of Suzhou New District

\section{Research Article}

Keywords: multi-omics, tuberculosis, zoonotic, lipid metabolism, transcriptome, signal transduction

Posted Date: February 16th, 2022

DOI: https://doi.org/10.21203/rs.3.rs-1301905/v1

License: (1) (1) This work is licensed under a Creative Commons Attribution 4.0 International License. Read Full License 


\section{Abstract}

The lipid composition performs important functions in interaction between macropha-ge and Mycobacterium tuberculosis (MTB)/Mycobacterium bovis (MB). Current understanding regarding the lipid responses of bovine alveolar macrophage (BAM) to MTB/MB is quite limiting. The present study conducted lipidomics and transcriptome to assess alterations in BAM lipid compositions upon $\mathrm{MB}$ and MTB infection. We found that both MTB and MB induced glycerophospholipids accumulation in BAM, and MTB induced more alterations in lipid composition. MTB could affect the contents of various lipids, especially ceramide phosphocholines, polystyrene(PS) (17:0/0:0), testolic acid and testosterone acetate. Meanwhile, MB particularly induced accumulation of 1-alkyl,2-acylglycerophosphoinositols. Both MB and MTB suppressed the contents of palmitoleamide, $\mathrm{N}$-ethyl arachidonoyl amine, $\mathrm{N}$-(1,1-dimethyl-2-hydroxyethyl) arachidonoyl I amine, eicosanoyl-EA, and PS (0-18:0/17:0) in BAM. Additionally, transcriptome analysis revealed that only MTB triggered genes involved in immune signaling and lipid-related pathways in BAM. And MTB mainly activated genes CXCL2 and CXCL3 relevant to NOD-like receptor, IL-17 and TNF to further induce lipid accumulation in BAM, which in turn promoted the formation of foam cells.

Altogether, the results of the present study provided evidence for changes inlipid metabolism of MTB/MBattacked BAM, and contributed towards the detection and treatment of zoonotic tuberculosis.

\section{Introduction}

Tuberculosis (TB) is a notorious disease caused by Mycobacterium tuberculosis (MTB) and Mycobacterium bovis (MB), which are known to severely affect a wide range of mammalian species, especially humans and cattle. In 2016, there were 10.4 million TB patients worldwide, and $~ 10 \%$ of TB cases were caused by MTB ${ }^{1}$. However, the application of currently available treatment strategies for TB is hampered owing to limited understanding regarding the host responses to MTB/MB and lack/absence of accurate clinical indicators. Importantly, species included in Mycobacterium tuberculosis complex (MTBC) are known to exhibit > 99\% genetic similarity, however, both MTB and MB have previously been shown to utilize distinct strategies to infect the host. Therefore, it is important to elucidate different and common responses of the host cells towards MB or MTB infections.

Macrophages are known to serve as important parasitic cells for MTB/MB colonization and development. In addition to this, they also perform important functions in innate immune response to defend/fight against MTB/MB infection ${ }^{2,3}$. Lipids providing energy are known to affect macrophage phenotype and function. Thus, disorder of lipid metabolism in macrophages would lead to a decrease in innate immune response associated with the occurrence of MTBC infection. The pathogenesis of tuberculosis is often accompanied by disorders of lipid metabolism ${ }^{4}$. Importantly, it was previously reported that MTB deployed lipids of macrophages as the main carbon source for survival and colonization, which further resulted in host susceptibility ${ }^{5}$. When attacked by MTB, the receptor CD36 present on the surface of macrophage membrane promoted lipid intake by macrophages, while the expression of lipid effluxation receptor was inhibited, which resulted in lipid accumulation and formation of foamy macrophages (FM) 
${ }^{6}$. Furthermore, previous studies provided evidences for relationship between lipid accumulation and formation of foamy macrophages (FM) during the process of MTB infection ${ }^{7}$. Interestingly, tumor necrosis factor-a (TNF-a) inhibited cholesterol metabolism and promoted lipid uptake in macrophages, further leading to macrophage foaming ${ }^{8}$. These foam macrophages would further secrete proinflammatory factors and metabolic enzymes to induce inflammation, which would suppress the host immune responses towards MTBC. Thus, elucidation of variations in lipid metabolism of macrophages, following MTB and MB infection would, contribute towards the prevention of tuberculosis occurrence.

Following engulfment by phagocytes, MTB and MB could trigger signals of intracellular pathogenassociated molecular patterns (PAMPs) ${ }^{9}$. Additionally, various signals involved in defense responses get activated, including signaling pathways relevant to TNF, NOD-like receptor, and Toll-like receptor ${ }^{10}$. Subsequently, these signals could invoke a bunch of immune responses, including autophagy, apoptosis, ROS burst, and inflammasome activation, to resist MTB/MB, ${ }^{11,12}$. It was previously shown that macrophages could further release TNF-a to form granulomas, which prevented MTBC growth via activation of TLRs signaling ${ }^{13}$. In addition to the critical role of pro-inflammatory cytokines (such as IFN$Y$, TNF-a, IL-6, and IL23), the presence of chemokines, such as CCL2, CCL3, and CXCL10, was also found to be imperative in defense response against MTB infection ${ }^{10}$. However, in case the pathogenic bacteria are not timely killed, the infection would be reactivated along with the failure of immune system, resulting in foaming of macrophages that is available for pathogen development.

The present study conducted integrated lipid metabolomics and transcriptome analyses on BAM to investigate/evaluate the changes in lipid metabolism of BAM, following MB and MTB infection. The study identified that the changes in lipid composition varied in BAM following MB and MTB infection. Additionally, transcriptome analysis showed that the occurrence of tuberculosis was generally associated with weakening of autophagy. Importantly, various genes relevant to signaling pathways, involved in innate immune response and lipid metabolism, were found to be specifically induced by MTB, but not MB. Altogether, the present integration analysis of metabolome and transcriptome of MTB/MB-attacked BAM elucidated basic strategies that could be utilized for the treatment and detection of tuberculosis.

\section{Materials And Methods}

\subsection{Bacterial culture strains}

The $M$. bovis strain MB was drug-sensitive and isolated from the lung tissues of cows with tuberculosis. All animal procedures at Animal and Plant Health Agency (APHA) were approved by the APHA Animal Welfare and Ethical Review Board (AWERB), in accordance with relevant guidelines and regulations, and all authors complied with the ARRIVE guidelines. The drug-sensitive M. tuberculosis strain was genotyped as MIRU-VNTR and isolated from the patient's sputum cultures at the People's Hospital of Suzhou New District. Written informed consent was obtained from all patients at the time of admission. The People's Hospital of Suzhou New District granted Ethical approval to carry out the study within its facilities. Both 
the strains were cultured to the mid-log phase in the Middlebrook 7H9 medium (Becton Dickinson and Company), as suggested by Gao et al. ${ }^{14}$. The M. bovis strain culture medium was further supplemented with $0.5 \%$ glycerol. The bacteria were cultured for 8 weeks for the infection experiment in a biosafety level-2 facility at the the People's Hospital of Suzhou New District (Su Zhou, China). Then, the bacteria were harvested from the medium through centrifugation at $3000 \mathrm{rpm}$ for $10 \mathrm{~min}$ following one washing in phosphate-buffered saline. To guarantee the same initial infection dose, the bacteria were resuspended in Dulbecco's Modified Eagle's Medium (DMEM) with 10\% fetal bovine serum (complete medium) to an OD600 of 1 equivalent to $3 \times 10^{8}$ bacteria/mL.

\subsection{Cell Collection, Culture, and Infection}

This study was approved by the ethics committee for use and care of animals at the Northwest A\&F University. Animal tissue samples were pooled and processed following the protocol guidelines recommended in the OIE Manual for Terrestrial Animals. Primary bovine alveolar macrophages (BAM) were collected from healthy male bovine lungs by pulmonary lung lavage via tracheal infusion of physiological saline solution as suggested by Romha et al. ${ }^{15}$. In total, $1 \mathrm{~L}$ of saline containing $4 \%$ antibiotic-antimycotic agent (100x) (SolarBio Life Science) was infused into the lungs via the bronchial tubes. The alveolar lavage fluid containing BAM was then collected. Approximately $4 \times 10^{7}$ total lung cells were cultured in the AIM VTM Medium (research grade, 12055091, Thermo Fisher) supplemented with $10 \%$ fetal bovine serum (FBS) and $4 \times$ antibiotics (Penicillin, Streptomycin, and Amphotericin B solution), following $y$-irradiation for $16 \mathrm{~h}$ at $37^{\circ} \mathrm{C}$ under $5 \% \mathrm{CO}_{2}$ before infection. BAM were infected with M. bovis strains or M. tuberculosis at a multiplicity of infection (MOI) of 10 bacilli per alveolar macrophage. Each group of cells from the treatment groups were infected with bacteria (10:1) at the MOI for $4 \mathrm{~h}$, respectively, at $38^{\circ} \mathrm{C}$ under $5 \% \mathrm{CO}_{2}$ atmosphere. We performed this experiment in triplicates for the uninfected control, MB-infected cells, and MTB-infected cells for $4 \mathrm{~h}$ of infection. In total, 9 samples were subjected to lipidomics. All MTB or MB infection experiments were conducted in the biosafety level-2 (bsl2) laboratory of The People's Hospital of Suzhou New District.

\subsection{Metabolites Extraction}

The samples of macrophages uninfected-and infected by MTB or MB with three replicates were collected for the lipidomic analyses. The samples were freeze-dried for $20 \mathrm{~h}$ at $-60^{\circ} \mathrm{C}$ and vacuum $\leq 5 \mathrm{~Pa}$, after which $10 \mathrm{mg}$ of the sample was collected in EP tube and dissolved in $200 \mu \mathrm{L}$ water. After $30 \mathrm{~s}$ of vortexing, the samples were homogenized at $35 \mathrm{~Hz}$ for $4 \mathrm{~min}$ and sonicated for $5 \mathrm{~min}$, twice. Subsequently, $480 \mu \mathrm{L}$ of the extract solution (MTBE: $\mathrm{MeOH}=5: 1$ ) was added, and the samples were further sonicated for $10 \mathrm{~min}$ on ice-water bath. Then, the samples were incubated at $-40^{\circ} \mathrm{C}$ for $1 \mathrm{~h}$ and centrifuged at $3000 \mathrm{rpm}$ for $15 \mathrm{~min}$ at $4^{\circ} \mathrm{C}$. Then, $300 \mu \mathrm{L}$ of the supernatant was transferred to a fresh tube in the vacuum. The dried samples were re-dissolved in $100 \mu \mathrm{L}$ of $50 \%$ methanol in dichloromethane through sonication. The constitution was then centrifuged at $13000 \mathrm{rpm}$ for $15 \mathrm{~min}$, and the supernatant was transferred to a glass vial for LC/MS analysis. Finally, the lipidomics of each experimental group with three biological replicates were determined. 


\subsection{LC-MS/MS Analysis}

The ExionLC Infinity series UHPLC System (AB Sciex) with the Kinetex C18 column $(2.1 * 100 \mathrm{~mm}, 1.7 \mu \mathrm{m}$; Phenomen) was used for LC/MS analyses. The mobile phase A consisted of $40 \%$ water and $60 \%$ acetonitrile with $10 \mathrm{mmol} / \mathrm{L}$ ammonium formate, and the mobile phase $B$ consisted of $10 \%$ acetonitrile and $90 \%$ isopropanol, with $10 \mathrm{mmol} / \mathrm{L}$ ammonium formate. The elution gradient was set to $0-12.0 \mathrm{~min}$, 40-100\% B; 12.0-13.5 min, 100\% B; 13.5-13.7 min, $100-40 \%$ B; $13.7-18.0 \mathrm{~min}, 40 \%$ B. The column temperature was set to $45^{\circ} \mathrm{C}$. The injection volume was $2 \mu \mathrm{L}$ (pos) and $6 \mu \mathrm{L}$ (neg), respectively.

The TripleTOF 5600 Mass Spectrometer was used to acquire the MS/MS spectra on an informationdependent basis (IDA) with the acquisition software (Analyst TF 1.7, AB Sciex) to evaluate the full scan survey MS data. The most intensive 12 precursor ions with intensity $>100$ were selected for MS/MS at collision energy (CE) of $45 \mathrm{eV}$. The ESI source conditions were set as follows: Gas 1 as 60 psi, Gas 2 as $60 \mathrm{psi}$, Curtain Gas as $30 \mathrm{psi}$, source temperature as $600^{\circ} \mathrm{C}$, declustering potential as $100 \mathrm{~V}$, and ion spray voltage floating (ISVF) as $5000 \mathrm{~V}$ or $-3800 \mathrm{~V}$ in positive or negative modes, respectively ${ }^{16}$.

\subsection{Data preprocessing and annotation}

An in-house program was developed using the R software for data analysis. The 'msconvert' program was used to convert the raw data files (.wiff format) to the mzXML format. Then, the mzxML files were loaded into LipidAnalyzer for data processing. The CentWave algorithm was applied for peak detection by $\mathrm{R}$ package XCMS. The lipid identification was achieved by in-house lipid library against their MS/MS spectrum ${ }^{17,18}$. We further used the R package metaboanalyst 3.0 to identify the pathway enrichment and categorize the differential lipid in each pairwise comparison.

\subsection{Collection of transcriptomic profiles of macrophage following MTB and MB attacks from GEO data repository}

As a publicly available transcriptome database, the Gene Expression Omnibus (GEO) contains series high throughput transcriptome profiles. To investigate the responses of macrophage to MTB and MB infection, 9 transcriptome datasets in the "fastq" form were downloaded, including the transcriptome profiles of macrophages to MTB and MB infections, whereas the transcriptome profiles of normal macrophage were set as control. Each group of profiles contained 3 replicates. The detailed descriptions of each transcriptome profile are shown in Table 1. 
Table 1

List of transcriptome profiles from the GEO database

\begin{tabular}{|lllll|}
\hline Run & BioSample & Experiment & GEO-Accession & Condition \\
\hline SRR6072076 & SAMN07693452 & SRX3213613 & GSM2792506 & MTB-infected \\
\hline SRR6072081 & SAMN07693447 & SRX3213618 & GSM2792511 & MTB-infected \\
\hline SRR7478850 & SAMN09623383 & SRX4348292 & GSM3259598 & Control \\
\hline SRR7478852 & SAMN09623381 & SRX4348294 & GSM3259600 & Control \\
\hline SRR7478851 & SAMN09623382 & SRX4348293 & GSM3259599 & MB-infected \\
SRR7478853 & SAMN09623380 & SRX4348295 & GSM3259601 & MB-infected \\
\hline
\end{tabular}

\subsection{Transcriptome analysis}

The reads in each transcriptome profile in the "fastq" form were aligned against the reference genome of Bos taurus (https://www.ncbi.nlm.nih.gov/assembly/GCF_002263795.1) by the software Tophat 2.1.0. Further assembly and quantification of genes were analyzed by software cufflinks, and the GFF file of Bos taurus was used as the reference. The raw Fragments Per Kilobase of transcript per Million (FPKM) data were further used to calculate the relative expression level. Genes matched the condition (log 2 Fold change $>1$; FDR $<0.05$ ) were regarded as the differentially expressed genes.

\subsection{Enrichment and protein-protein interaction network analysis}

R packages clusterprofiler was applied to analyze gene ontology (GO) and KEGG pathway enrichment analyses on differential genes. A two-tailed Fisher's exact test was used to test the significance of each $\mathrm{GO}$ term or the pathway. The pathway and GO terms with $\mathrm{p}<0.05$ was recognized as significant categorization. The candidate differential genes were submitted to the STRING database to construct the protein-protein interaction network. The interaction relationship was considered only between the candidate genes, so that the genes not involved in the network were excluded.

\section{Results}

\subsection{MTB- and MB-infection Induced Significant Alterations in Lipid Metabolism of BAM.}

To assess the impact of MB- or MTB-infection on lipid composition of BAM, the present study compared the lipidomics profiles produced by normal BAM and MTB- or MB-infected BAM. It was observed that both pathogenic bacteria (MB and MTB) induced distinct changes in lipid composition of BAM. When compared with normal BAM (CK), 47 differential lipids were produced 4 hours post inoculation (hpi) of $\mathrm{MB}$, wherein 19 lipids were up-regulated and 28 lipids were down-regulated lipids (Fig. 1B, $\log 1.2 \mathrm{fc}<-1$ 
or $>1, P<0.05)$. Similarly, 98 differentially expressed metabolites were identified in MTB vs. CK group. Importantly, the contents of 79 lipids were found to be increased, while 19 lipids were decreased in MTBinfected BAM (Fig. 1C). For MB vs. MTB comparison, 110 different lipids were identified, 33 of these lipids were up-regulated, whereas 77 lipids were down-regulated (Fig. 1D). Following this, Principle Components Analysis (PCA) was used to get an immediate overview regarding the patterns in the data and assess whether these patterns were associated with MTB or MB infection (Fig. 1A). Importantly, PCA plots showed a projection of the data in two-dimensional space, such that the maximal spread of the patterns related to MTB or MB infection were visible, which indicated that lipid metabolomics of MTB- and MBattacked BAM exhibited significantly different characteristics. Indeed, the two score plots of the PCA models showed a clear separation between each experimental group (Fig. 1A), which indicated that MTB or MB induced significant changes in BAM lipid composition. The top two components, represented by principal component (PC) 1 and PC 2, explained $29.2 \%$ and $22.8 \%$ of the observed variance/variations, respectively. Since PCA is an unsupervised method and MB- and MTB-infection were not taken into account in while defining the components, the patterns shown in the first two PCs were the ones with globally most conspicuous characteristics. These results suggested that both MTB and MB induced significant alterations in BAM lipid composition. Meanwhile, when compared with MB-infected BAM, more significant differences were induced in BAM lipid composition by MTB-infection.

\subsection{MTB- and MB-infection Differentially Altered Lipid Metabolism of BAM.}

The results for PCA revealed a high correlation of MB- and MTB-infection with specific lipid profile. However, in general, it would be better to consider supervised approaches to specifically focus on relevant differences between MB- and MTB-infection. Therefore, PLS-DA module was established for better identification of the differences in lipidomics of MB- and MTB-infected BAM, and VIP values were obtained to further assess the significance of each lipid (Fig. 2C). Subsequently, three sets of differentially expressed lipids were identified from MB vs. MTB, MB vs. CK, and MTB vs. CK pairwise comparisons, and the compounds from PLS-DA module were used to distinguish key compounds (Fig. 2A and Fig. 2D). In particular, seven intersections were obtained, which included 173 lipid compounds, with VIP $>1.5, P<0.05$, and fold change $>1$.2. It was observed that these lipids mainly clustered into three modules, based on their relative concentration in each experimental group (Fig. 2B). The contents of most lipids were recorded to be higher in MTB-infected BAM, whereas the differences in the contents of all these lipids were fairly minor between control and MB-infected BAM (Fig. 2D).

On the basis of VIP value of PLS-DA module, it was observed that all the top 15 lipid compounds, which included palmitoleamide, 3ß-(3-methyl-butanoyloxy)-villanovane-13a,17-diol, PS (17:0/0:0), HexCer-NDS d19:0/31:1, N-ethyl arachidonoyl amine, GlcADG 18:0-17:1, testosterone acetate, $\mathrm{N}$-(1,1-dimethyl-2hydroxy-ethyl) arachidonoyl amine, PE 22:5e/18:2, and HBMP 16:0-16:1-16:1, were dramatically altered in MTB- or MB-infected BAM. However, the levels of these metabolites were not highly increased under MB-infection (Fig. 2C and D). Among these 15 lipids, the contents of palmitoleamide, $\mathrm{N}$-ethyl 
arachidonoyl amine, $\mathrm{N}$ - (1,1-dimethyl-2-hydroxy-ethyl) arachidonoyl amine, eicosanoyl-EA, and PS (018:0/17:0) were found to be commonly suppressed during MTB- and MB-infection (Fig. 2D). Meanwhile, ten lipids, including PE 22:5e/18:2, PS (17:0/0:0), 33-(3-methyl-butanoyloxy)-villanovane-13a,17-diol, testolic acid, HexCer-NDS d19:0/31:1, testosterone acetate, PEtOH 27:0-17:1, TAG 16:0-16:0-21:4, GICADG 18:0-17:1, and HBMP 16:0-16:1-16:1 were found to be increased by MTB (Fig. 2D). Typically, no increase in the contents of these 15 lipids was observed in MB-infected BAM samples (Fig. 2D). All these results reached a credible consensus with PCA plots (Fig. 1A), and indicated that MTB infection induced more significant alterations in BAM lipid composition, as compared to MB-infection.

\subsection{Pathway Enrichment Analysis on Increased Metabolites in MTB- and MB-infected BAM.}

As shown in Fig. 3, a detailed heatmap clustering was constructed for the lipids with VIP $>1.0, F C>1.2$, and $P<0.05$, to clearly analyze the alterations in the contents of these key lipids in MTB- and MB-infected BAM. It was observed that the levels of all these 86 lipids (72 in MTB vs. CK and 14 in MB vs. CK) were higher in MTB-infected BAM as compared to normal BAM (Fig. 3A and B). Importantly, only 27 of these lipids were found to be highly increased in MB-infected BAM (Fig. 3A and B).

Subsequently, a pathway enrichment analysis was performed on these two sets of lipids against KEGG database. The results showed that glycerolipid metabolism, phosphodylinositol signaling system, and glycerophospholipid metabolism were invoked in MB-challenged BAM (Fig. 3C). At the same time, MTB infection could induce alterations in linoleic acid metabolism, a-linolipid metabolism, sphingolipid metabolism, arachidonic acid metabolism, and glycerophospholipid metabolism. It was observed that both MTB and MB affected the pattern of glycophospholipid metabolism pathway. It has been previously reported that glycophospholipid metabolism was involved in energy conversion of host ${ }^{19}$. Therefore, it was proposed that MTB and MB could induce an increase in glycophospholipid, which would serve as a nutrient for the development and colonization.

\subsection{Classification analysis of MTB- and MB-induced lipids.}

To further explicit the detailed lipid categorizes that were increased in MTB-infected BAM, a classification enrichment analysis was performed on the compounds accumulated in MTB-infected BAM. For the categorization based on super-class level, it was observed that MTB-infection mainly invoked the accumulation of glycerophospholipids. Importantly, half of the lipids belonged to this category (Fig. 4B). The other lipids were mainly categorized into classes of fatty acids, sterol lipids, and sphingolipids. The lipids relevant to prenol lipids and polyketides class constituted a relatively small percentage of all lipids induced by MTB-infection (Fig. 4B). For the categorization of main-class level, it was observed that MTBinduced lipids relevant to glycerophospholipids mainly belonged to glycerophosphoinositols, glycerophosphates, and glycerophosphoiglycerol classes. At the same time, various other classes were also found to be significantly enriched, which included sterols, fatty acyl glycosides, sphingolipid bases, and steroids classes, with $P<0.05$, (Fig. 4D). Various previous studies indicated that sterol-related lipids 
represent the dominant category, whose metabolism was dramatically altered by MTB-infection 7,20. Further, in terms of sub-class categorization, it was observed that lipid-relevant to ceramide phosphocholines, FA glycosides 1-alkyl,2-acylgly, N-acylsphingosines, and monoacylglycerol phosphoglycerols were highly enriched in MTB-induced lipid metabolism network (Table 2). 
Table 2

Lipid Sub-classification of MTB- or MB infection

\begin{tabular}{|c|c|c|c|}
\hline & Metabolite Set & Metabolites Number & $P$ value \\
\hline \multirow[t]{15}{*}{ MTB Infection } & 1-alkyl,2-acylglycerophosphoinositols & 3 & $3.22 \mathrm{E}-07$ \\
\hline & 1-alkyl,2-acylglycerophosphates & 2 & 8.07E-05 \\
\hline & $\mathrm{N}$-acylsphingosines & 2 & $9.18 \mathrm{E}-05$ \\
\hline & C19 steroids & 2 & $2.15 \mathrm{E}-04$ \\
\hline & Diacylglycerophosphoglycerols & 3 & 0.00111 \\
\hline & Diacylglycerophosphoinositols & 3 & 0.00118 \\
\hline & Ceramide phosphocholines & 1 & 0.00173 \\
\hline & FA glycosides & 1 & 0.00238 \\
\hline & Monoacylglycerophosphoserines & 1 & 0.0067 \\
\hline & Monoacylglycerophosphoglycerols & 1 & 0.0067 \\
\hline & Saturated Fatty Acids & 1 & 0.00821 \\
\hline & Cycloartanols & 1 & 0.0112 \\
\hline & 1-alkyl,2-acylglycerophosphoserines & 1 & 0.0129 \\
\hline & LPE & 1 & 0.0144 \\
\hline & Sphingoid base analogs & 1 & 0.0157 \\
\hline \multirow[t]{9}{*}{ MB Infection } & 1-alkyl,2-acylglycerophosphoinositols & 2 & $3.24 \mathrm{E}-06$ \\
\hline & 1-alkyl,2-acylglycerophosphates & 1 & 0.00272 \\
\hline & Bile acids, alcohols and derivatives & 1 & 0.0029 \\
\hline & C19 steroids & 1 & 0.00444 \\
\hline & C24 bile acids & 1 & 0.00679 \\
\hline & Diacylglycerophosphates & 1 & 0.0398 \\
\hline & Wax monoesters & 1 & 0.0423 \\
\hline & Diacylglycerophosphoinositols & 1 & 0.0424 \\
\hline & Flavones & 1 & 0.126 \\
\hline
\end{tabular}

Subsequently, the study further classified the lipids induced by MB. The results for super-class level showed that glycerophospholipids still represented the main family that was induced by both $\mathrm{MB}$ and MTB (Fig. 4A and B). Therefore, it was speculated that alterations in glycerophospholipids was a 
common response of macrophages towards MB and MTB infection. Among the main class, lipids belonging to glycerophosphoinositols, glyophosphates, and steroids were the major compounds that were associated with MB-infection (Fig. 4C). It has been previously reported that sterol-related lipids could be activated by MB-infection in BAM ${ }^{19}$. Finally, for sub-class level, only 1 -alkyl,2acylglycerophosphoinositols were found to be highly enriched under MB-infection (Table 2).

Therefore, the present study revealed that MTB could induce alterations in the contents of ceramide phosphocholines, FA glycosides 1-alkyl,2-acylgly, and N-acylsphingosines, whereas MB induced accumulation of 1-alkyl,2-acylglycerophosphoinositols. These lipids might function as specific energy source for the development of both these pathogenic bacteria in vivo, and thus could be used as clinical index/indices for the detection of pathogenic MTB/MB associated with tuberculosis.

\subsection{Genes relevant to signaling were dramatically altered by MTB and MB infection.}

To further elucidate BAM responses to both MTB and MB infection, three sets of transcriptomic profiles with two replicates were obtained from GEO database and analyzed. In particular, transcriptomic data for MTB- and MB-infected BAM were compared with that of normal BAM. For MB vs. CK comparison, 698 and 362 genes were found to be up- and down-regulated, respectively, by MB attacks (Fig. 5A). For MTB vs. CK comparison, 411 genes were up-regulated and 344 genes were down-regulated (Fig. 5C). To clearly understand the function of these genes, GO enrichment analysis was performed on the genes associated with MTB or MB attacks, and the combined functional assignments are provided in Fig. 5 . The results of the analyses revealed that the genes that were up- and down-regulated genes under MB-infection were primarily enriched for "enzyme regulator activity", "regulation of response to stimulus", "regulation of signal transduction", and "regulation of immune system process" (Fig. 5B). This further suggested that MB infection could stimulate the activation of innate immune system of BAM. Furthermore, it was observed that various $\mathrm{GO}$ terms relevant to signaling and immune system/response were significantly enriched among the genes associated with MTB-infection, which included "cytokine activity", "signaling receptor binding", "calmodulin binding", "defense response", "inflammatory response", and "immune response" (Fig. 5D). Remarkably, cytokine-related terms represented the main alterations observed in BAM in response to MTB. Cytokines, such as TNF-a, IFN-y, IL-6, IL-12, IL-23/IL17, and others, are known

to be involved in inflammation. In fact, these molecules play important roles in BAM lipid metabolism ${ }^{10}$. Based on these results, it was concluded that MTB induced more dramatic alterations in innate immune response of BAM.

\subsection{MTB infection resulted in alterations in lipid-related metabolisms in BAM.}

To gain detailed insights into the differences in BAM alterations induced by MTB and MB, differentially expressed genes from MB- or MTB-infected and control groups were further annotated by KEGG pathway. The genes affected by MB were mainly enriched in Focal adhesion, Rap1 signaling pathway, MAPK 
signaling pathway, RAS signaling pathway, and PI3K-Akt signaling pathway (Table S3). Subsequently, pathway analysis for MTB-affected genes showed that these genes mainly functioned in signaling pathways relevant to TNF, NOD-like receptor, NF-KB, IL-17, MAPK, RAS, and Toll-like receptor (Table S3). Both MTB and MB could influence MAPK and RAS signaling pathways, which are important parts of innate immune system. It was further observed that MTB could significantly affect fatty acid-related pathways, which were not identified in MB vs. CK comparison (Table S3). Thus, series of genes that were only activated or suppressed by MTB infection were obtained and pathway analysis was performed (Fig. 6A). The results showed that various genes involved in lipid metabolism were only suppressed to down-regulate MTB attacks, whereas the genes involved in immune-related signaling pathways, such as signaling pathway of TNF, NOD-like receptor, and NF-KB, were highly activated in MTB-challenged BAM (Fig. 6B and D). Moreover, the construction of protein interaction network for these lipid- and signalingrelated genes revealed that MTB could result in lipid accumulation in BAM that was mediated via activation of CXCL2 and CXCL3, which were further involved in signaling pathway of NOD-like receptor, IL-17, and TNF (Fig. 6C). Altogether, these results provided suitable explanation for the metabolic phenomena involved in MTB infection, wherein MTB-infection induced the accumulation of various lipid compounds and altered signaling in BAM. Importantly, MB-infection could not result in such dramatic mechanism.

\section{Discussion}

The main characteristics of MTB and MB pathogenicity primarily involved MTB and MB induced alterations in lipid metabolism to obtain nutrients for their development and colonization in macrophages. In response to this, macrophages could be polarized to M1 type to resist pathogen infection, mediated via activation of lipopolysaccharide (LPS) and Toll-like receptor (TLR) signaling ${ }^{21}$. In the present study, it was observed that MTB induced more alterations in BAM lipid composition as compared to MB infection. Various lipids, especially glycerophospholipids, were found to be increased in BAM following MTB and MB infection, and thus it was concluded that glycerophospholipids could serve as clinical indicators for tuberculosis detection. Importantly, MTB also induced alterations in the contents of ceramide phosphocholines, FA glycosides 1-alkyl,2-acylgly, and N-acylsphingosines, whereas MB only induced accumulation of 1-alkyl,2-acylglycerophosphoinositols. Furthermore, MTB dramatically invoked the expression of genes involved in various immune signaling- and lipid-related pathways, including TNF signaling pathway, NOD-like receptor signaling pathway, Toll-like receptor signaling pathway, and fatty acid metabolism. However, MB attacks did not induce dramatic activation of signaling pathways or lipidrelated metabolism. In consensus with aforementioned metabolomics results, these phenomena suggested that MTB induced more alterations in BAM lipid metabolism. Therefore, the present study provided in-depth/deep insights into different mechanisms of defense responses elicited in BAM against MTB and MB, which would contribute towards the detection and treatment of human and cattle tuberculosis. 
Glycerophospholipids are known to play an important role in the maintenance of cell membrane stability and signal transduction in host cells ${ }^{22}$. The results of the present study showed that MTB and MB attacks induced an increase in the contents of glycerophospholipids, suggesting that both these microbes might destroy macrophage membrane structure, while host macrophages could invoke intracellular lipid-related signal to activate defense responses. Besides this, glycerophospholipids are also known to play important roles in energy conversion, vesicle formation, and transmembrane transport ${ }^{19}$. Thus, glycerophospholipids could serve as a nutrient for the host cell or MTB/MB. Additionally, polykelides, one of the upregulated categories, have been previously shown to exhibit anti-bacterial and anti-parasitic functions. Thus, an increase in these compounds would contribute towards macrophage defense against MTB/MB. Sterol lipids are important components of cell membrane structure, which are known to play a positive regulatory role in resisting pathogen infection ${ }^{23}$. Consequently, an increase in these lipids would strengthen the host resistance towards MTB/MB attacks. Importantly, it has been previously reported that the levels of sphingolipids were increased in macrophages during MTB infection. Moreover, sphingolipids have been previously shown to be involved in host immune system, which could further induce inflammatory response in macrophages to strengthen host resistance. ${ }^{24}$. It was previously reported that MTB infection primarily affected lipid metabolism of macrophages for successful development and colonization ${ }^{25}$. The present data showed an increase in the content of glycerolipids in MTB/MB-attacked macrophages. Similarly, it has been previously established that MTB induced accumulation of glycerolipids and liposomes as nutrient, for its development in macrophages ${ }^{26}$. Meanwhile, MTB induced the accumulation of lipids mainly via alteration of glycolytic pathway to ketone body synthesis ${ }^{27}$. Thus, disorder of lipid metabolism in macrophages would contribute towards tuberculosis occurrence ${ }^{28}$. Additionally, MTB could further stimulate the accumulation of tumor necrosis factor-a (TNF-a) to suppress cholesterol metabolism in macrophages and increase lipid intake, resulting in the formation of $\mathrm{FM}^{8}$. The present study reported dramatic activation of TNF-a signaling pathway in MTB-infected macrophages, which provided suitable explanation for the pathogenesis of MTB. Importantly, accumulation of lipids could further inhibit autophagy-related pathways, resulting in the formation of FM, which weakened the phagocytosis of MTB/MB and inhibited their proliferation 29,30. The present study identified that increased lipids 1-alkyl and 2-acylglycerophosphoinositols, 1-alkyl and 2-acylglycerophosphates, C19 steroids, and diacylglycerophosphoinositols provided convenience for MB and MTB colonization in macrophages. In fact, these lipids could be used as clinical indicators for tuberculosis.

The phenomenon that more defense-related responses were activated during MTB-attacks supported the current hypothesis that bovine macrophages were more sensitive to MTB than MB. Among these defense responses, various signaling-related pathways, including pathways relevant to NF-K B signaling, IL-17 signaling, MAPK signaling, RAS signaling, and Toll-like receptor signaling, were activated by both MTB and MB attacks. Importantly, only RAP1, MAPK, RAS, and PI3K-Akt signaling pathways were activated by $M B$ attacks, which suggested that MB could evade the host immune system by certain strategies. Signaling-related pathways are known to be important parts of immune system of the host that are 
involved in defense against pathogen infection ${ }^{31}$. Activation of MAPK signaling pathway is known to be inevitable for host response towards pathogens. In particular, it is important for the activation of immunomodulatory molecules, such as TNF- $a$ and IL, which have been previously shown to be important for macrophages to defend/fight MTB invasion ${ }^{25}$. The present study also reported activation of NF-KB, IL-17, and Toll-like receptor signaling pathways in MTB-attacked macrophages. These pathways are known to be closely associated with host defense responses, such as various pro-inflammatory responses and release of antimicrobial effectors ${ }^{32}$. Additionally, activation of IL-17 signaling pathway has been identified in MTB-infected macrophages, and IL-17 could regulate pro-inflammatory response and chemokines ${ }^{10,33}$. Moreover, activation of peripheral inflammatory cells could produce microbiocidal substances, such as TNF-a, to inhibit pathogenic bacteria and protect the host cells ${ }^{13}$. Besides this, it has been previously reported that MTB-induced IFN-ץ, IL-6, IL-12, IL-23/IL17, and other cytokines that performed important functions in response towards MTB, and promoted inflammation ${ }^{10}$. The activation of chemokines could further accelerate the migration of different cell subsets to MTB infected tissues and promote granulomatous formation to kill MTB ${ }^{34,35}$. The present study identified a bunch of MTBinduced chemokines in macrophages, which included CCL2, CCL3, CCL5, CCL7, CCL12, CXCL2, CXCL8, and CXCL10. These chemokines were involved in pro-inflammatory response and inhibited MTB. Importantly, protein interaction network showed that CXCL2 and CXCL3 could regulate lipid metabolisms by directly interacting with lipid-related genes that were associated with MTB infection. Therefore, the study highlighted the role/involvement of chemokines in the interaction mechanisms of MTB and macrophages.

Altogether, the presented integration analysis of transcriptome and lipid metabolomics on BAM and $M T B / M B$ revealed the involvement of different mechanisms in the interaction between BAM and MTB/MB. The study proved that disorder of lipid metabolism in BAM, which resulted in the FM formation, was associated with the pathogenesis of MTB and MB. Importantly, BAM could deploy a series of signaling pathways and chemokines to defend/fight against MTB infection, whereas MB could evade only a part of BAM defensive responses. These results provided deeper insights into different defense responses of BAM towards MTB or MB infections, which would further assist in the development of novel strategies for the treatment and detection of tuberculosis in the future.

\section{Declarations}

\section{Availability of materials and data}

All data generated or analysed during this study are included in this published article [and its supplementary information files].

\section{Conflict of interest statement}

All other authors declare no competing interests. 


\section{Acknowledgements}

The authors would like to thank all the reviewers who participated in the review, as well as MJ Editor (www.mjeditor.com) for providing English editing services during the preparation of this manuscript.

\section{References}

1. Waters, W.R., \& Palmer, M.V. Mycobacterium bovis Infection of Cattle and White-Tailed Deer: Translational Research of Relevance to Human Tuberculosis. ILAR J. 56, 26-43. https://doi.org/10.1093/ilar/ilv001 (2015).

2. Rienksma, R.A., Schaap, P.J., Martins Dos Santos, V.A.P., \& Suarez-Diez, M. Modeling Host-Pathogen Interaction to Elucidate the Metabolic Drug Response of Intracellular Mycobacterium tuberculosis. Front. Cell Infect. Microbiol. 9, 144. https://doi.org/10.3389/fcimb.2019.00144 (2019).

3. Lu, Y., et al. Effect of gap junctions on RAW264.7 macrophages infected with H37Rv.Medicine (Baltimore). 97, e12125. https://doi.org/10.1097/MD.0000000000012125 (2018).

4. Wipperman, M.F., Sampson, N.S., \& Thomas, S.T. Pathogen roid rage: cholesterol utilization by Mycobacterium tuberculosis. Crit. Rev Biochem Mol Biol. 49, 269-293. https://doi.org/10.3109/10409238.2014.895700 (2014).

5. Lee, W., VanderVen, B.C., Fahey, R.J., \& Russell, D.G. Intracellular Mycobacterium tuberculosis exploits host-derived fatty acids to limit metabolic stress.J Biol Chem. 288, 6788-6800. https://doi.org/10.1074/jbc.M112.445056 (2013).

6. Chistiakov, D.A., Melnichenko, A.A., Myasoedova, V.A., Grechko, A.V., \& Orekhov, A.N. Mechanisms of foam cell formation in atherosclerosis.J Mol Med (Berl). 95, 1153-1165. https://doi.org/10.1007/s00109-017-1575-8 (2017).

7. Kim, M.J., et al. Caseation of human tuberculosis granulomas correlates with elevated host lipid metabolism. EMBO Mol Med. 2, 258-274. https://doi.org/10.1002/emmm.201000079 (2010).

8. Ma, A.Z., Zhang, Q., \& Song, Z.Y. TNFa alter cholesterol metabolism in human macrophages via PKCtheta-dependent pathway.BMC Biochem. 14, 20. https://doi.org/10.1186/1471-2091-14-20 (2013).

9. Kaur, D., Guerin, M.E., Skovierova, H., Brennan, P.J., \& Jackson, M. Chapter 2: Biogenesis of the cell wall and other glycoconjugates of Mycobacterium tuberculosis.Adv Appl Microbiol. 69, 23-78. https://doi.org/10.1016/S0065-2164(09)69002-X (2009).

10. Zuniga, J., et al. Cellular and humoral mechanisms involved in the control of tuberculosis. Clin Dev Immunol. 2012, 193923. https://doi.org/10.1155/2012/193923 (2012).

11. Bain, J.R., et al. Metabolomics applied to diabetes research: moving from information to knowledge.Diabetes. 58, 2429-2443. https://doi.org/10.2337/db09-0580 (2009).

12. Guasch-Ferre, M., et al. Metabolomics in Prediabetes and Diabetes: A Systematic Review and Metaanalysis.Diabetes Care. 39, 833-846. https://doi.org/10.2337/dc15-2251 (2016). 
13. Takeda, K., \& Akira, S. Toll-like receptors in innate immunity.Int Immunol. 17, 1-14. https://doi.org/10.1093/intimm/dxh186 (2005).

14. Gao, W., et al. Lipidomics revealed the global lipid responses of primary bovine alveolar macrophages to infections of Mycobacterium tuberculosis and Mycobacterium bovis. Int Immunopharmacol. 108407. https://doi.org/10.1016/j.intimp.2021.108407 (2021).

15. Romha, G., Gebru, G., Asefa, A., \& Mamo, G. Epidemiology of Mycobacterium bovis and Mycobacterium tuberculosis in animals: Transmission dynamics and control challenges of zoonotic TB in Ethiopia.Prev Vet Med. 158, 1-17. https://doi.org/10.1016/j.prevetmed.2018.06.012 (2018).

16. Want, E.J., et al. Global metabolic profiling procedures for urine using UPLC-MS.Nat Protoc. 5, 10051018. https://doi.org/10.1038/nprot.2010.50 (2010).

17. Kuhl, C., Tautenhahn, R., Bottcher, C., Larson, T.R., \& Neumann, S. CAMERA: an integrated strategy for compound spectra extraction and annotation of liquid chromatography/mass spectrometry data sets.Anal Chem. 84, 283-289. https://doi.org/10.1021/ac202450g (2012).

18. Lam, S.M., Tian, H., \& Shui, G. Lipidomics, en route to accurate quantitation. Biochim Biophys Acta Mol Cell Biol Lipids. 1862, 752-761. https://doi.org/10.1016/j.bbalip.2017.02.008 (2017).

19. Van Meer, G., \& de Kroon, A.I. Lipid map of the mammalian cell.J Cell Sci. 124, 5-8. https://doi.org/10.1242/jcs.071233 (2011).

20. Ahluwalia, P.K., Pandey, R.K., Sehajpal, P.K., \& Prajapati, V.K. Perturbed microRNA Expression by Mycobacterium tuberculosis Promotes Macrophage Polarization Leading to Pro-survival Foam Cell.Front Immunol. 8, 107. https://doi.org/10.3389/fimmu.2017.00107 (2017).

21. Murray, P.J., et al. Macrophage activation and polarization: nomenclature and experimental guidelines.Immunity. 41, 14-20. https://doi.org/10.1016/j.immuni.2014.06.008 (2014).

22. Feillet-Coudray, C., Fouret, G., Casas, F., \& Coudray, C. Impact of high dietary lipid intake and related metabolic disorders on the abundance and acyl composition of the unique mitochondrial phospholipid, cardiolipin.J Bioenerg Biomembr. 46, 447-457. https://doi.org/10.1007/s10863-0149555-y (2014).

23. Zhang, C., et al. Lipid metabolism in inflammation-related diseases.Analyst. 143, 4526-4536. https://doi.org/10.1039/c8an01046c (2018).

24. Nagahashi, M., et al. Targeting the SphK1/S1P/S1PR1 Axis That Links Obesity, Chronic Inflammation, and Breast Cancer Metastasis.Cancer Res. 78, 1713-1725. https://doi.org/10.1158/0008-5472.CAN-17-1423 (2018).

25. Shukla, S.K., et al. Pathway analysis of differentially expressed genes in Mycobacterium bovis challenged bovine macrophages. Microb Pathog. 115, 343-352. https://doi.org/10.1016/j.micpath.2017.11.065 (2018).

26. Russell, D.G., Cardona, P.J., Kim, M.J., Allain, S., \& Altare, F. Foamy macrophages and the progression of the human tuberculosis granuloma.Nat Immunol. 10, 943-948. https://doi.org/10.1038/ni.1781 (2009). 
27. Singh, V., et al. Mycobacterium tuberculosis-driven targeted recalibration of macrophage lipid homeostasis promotes the foamy phenotype. Cell Host Microbe. 12, 669-681. https://doi.org/10.1016/j.chom.2012.09.012 (2012).

28. Lao, W., et al. Evaluation of the relationship between MARCO and CD36 single-nucleotide polymorphisms and susceptibility to pulmonary tuberculosis in a Chinese Han population.BMC Infect Dis. 17, 488. https://doi.org/10.1186/s12879-017-2595-2 (2017).

29. Krause, B.R., et al. In vivo evidence that the lipid-regulating activity of the ACAT inhibitor Cl-976 in rats is due to inhibition of both intestinal and liver ACAT.J Lipid Res. 34, 279-294. (1993).

30. Genoula, M., et al. Formation of Foamy Macrophages by Tuberculous Pleural Effusions Is Triggered by the Interleukin-10/Signal Transducer and Activator of Transcription 3 Axis through ACAT Upregulation.Front Immunol. 9, 459. https://doi.org/10.3389/fimmu.2018.00459 (2018).

31. Roskoski, R., Jr. Corrigendum to "Targeting ERK1/2 protein-serine/threonine kinases in human cancers" [Pharmcol. Res. 142 (2019) 151-168].Pharmacol Res. 143, 206. https://doi.org/10.1016/j.phrs.2019.03.019 (2019).

32. Liu, S., et al. Recombinant Mtb9.8 of Mycobacterium bovis stimulates TNF-alpha and IL-1beta secretion by RAW264.7 macrophages through activation of NF-kappaB pathway via TLR2.Sci Rep. 8, 1928. https://doi.org/10.1038/s41598-018-20433-x (2018).

33. Jurado, J.O., et al. IL-17 and IFN-gamma expression in lymphocytes from patients with active tuberculosis correlates with the severity of the disease.J Leukoc Biol. 91, 991-1002. https://doi.org/10.1189/jlb.1211619 (2012).

34. Algood, H.M., Chan, J., \& Flynn, J.L. Chemokines and tuberculosis. Cytokine Growth Factor Rev. 14, 467-477. https://doi.org/10.1016/s1359-6101(03)00054-6 (2003).

35. Saunders, B.M., \& Britton, W.J. Life and death in the granuloma: immunopathology of tuberculosis.Immunol Cel/ Biol. 85, 103-111. https://doi.org/10.1038/sj.icb.7100027 (2007).

\section{Figures}



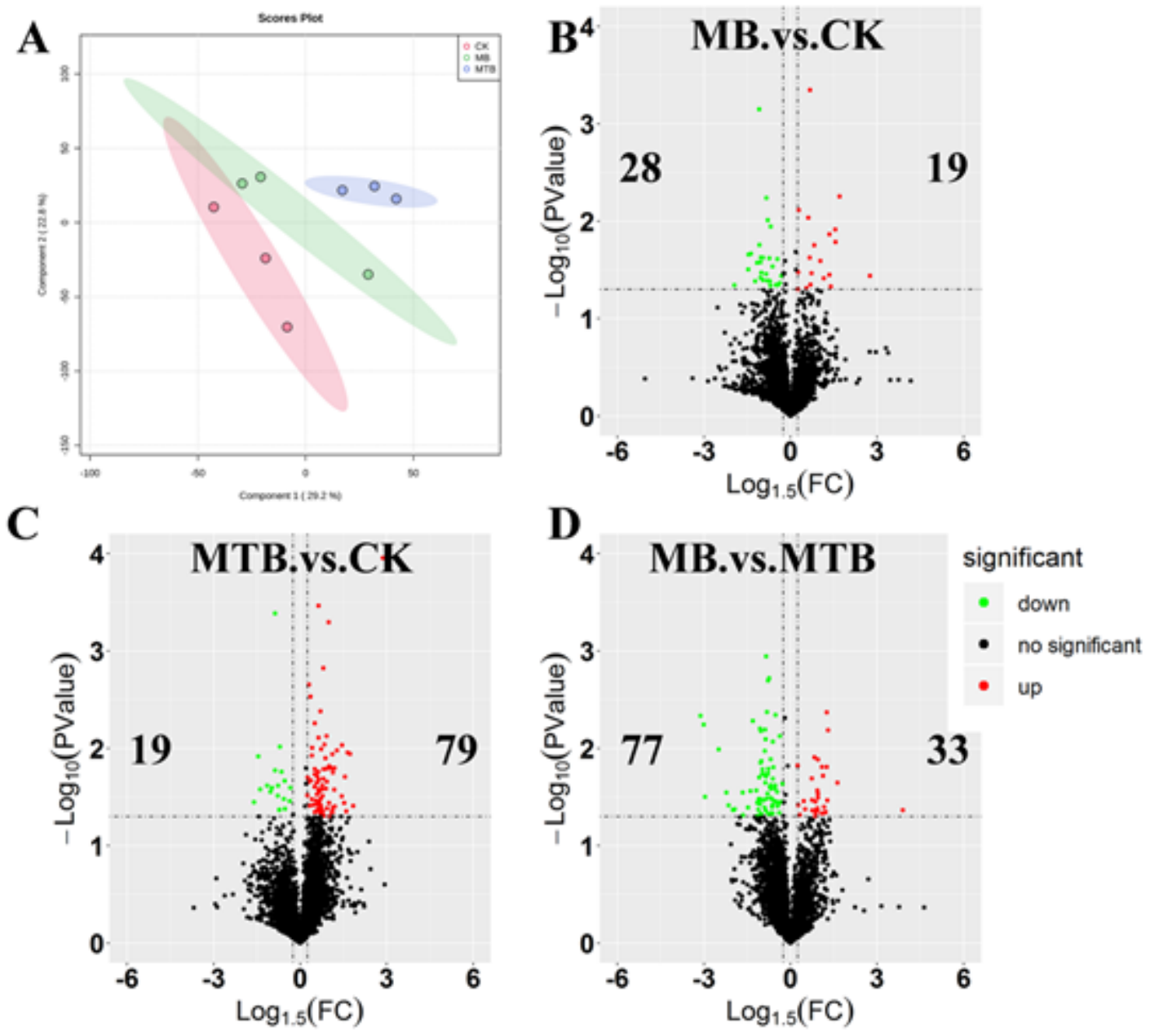

Figure 1

Overview of the alteration of lipid metabolisms of BAM following MTB and MB attacks. A: Principal component analysis represents the degree of separation of all samples and the stability between reproductions of each sample groups. Each circle represents a sample, the circles labeled by the same color indicates the samples with the same treatment. B, C, D: Volcano plots of content alterations of lipids in each pairwise comparison (B: MB. vs.CK; C: MTB. vs. CK; D: MB. vs. MTB). The gray points are lipids without significant alteration. The upregulated and downregulated lipids (|Log1.2(Foldchange) $\mid>1.0 ; \mathrm{P}<$ 0.05 ) are labeled by red and green colors, respectively. 


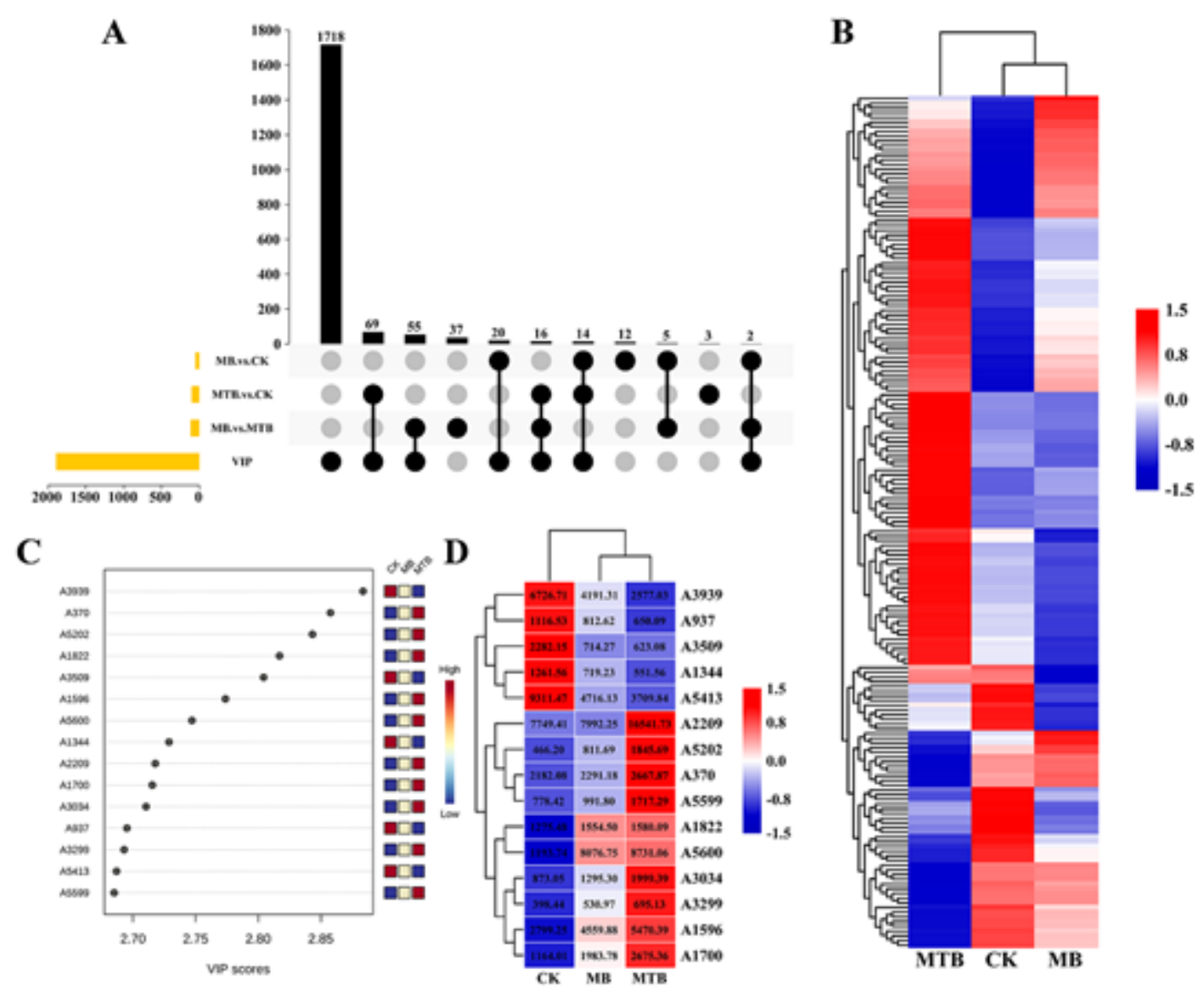

Figure 2

Legend not included with this version 

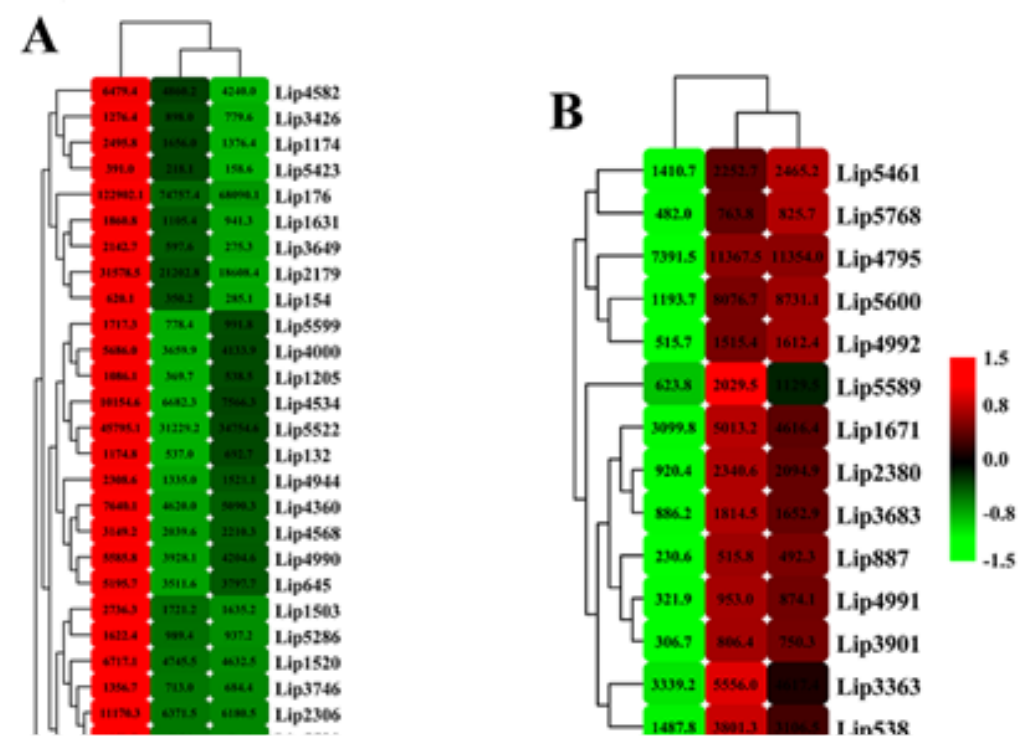

\section{Figure 3}

Pathway enrichment analysis on MTB- and MB-induced lipids. A, B: Relative abundance of lipids associated with the MTB or MB attacks. Green and red colors indicate metabolites with relative low and high abundance, respectively (A: MTB-induced lipids; B: MB-induced lipids). C: Pathways associated with $M B$ and MTB infection. Blue represents MB-induced pathways, while red represents MTB-induced ones ( $P$ $\llbracket 0.05)$. 


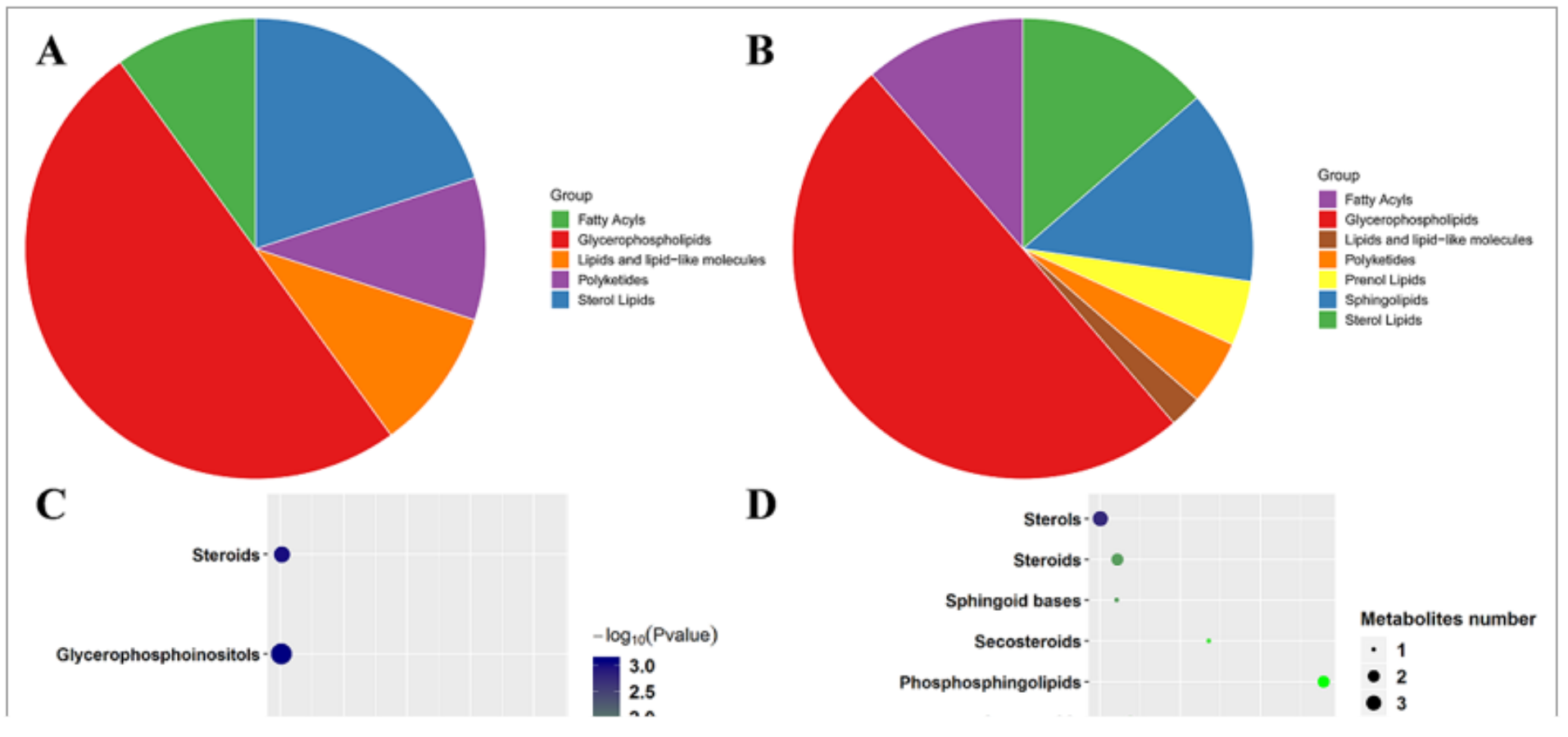

Figure 4

Classification of MTB- and MB-induced lipids. A, B: The pie chart represents the main lipid super-classes associated with MTB- $(B)$ and MB-infection (A). The area represents the percentage of each class. C, D: Lipid main class enrichment analysis under MTB (B) and MB (A) attacks, respectively. The size of circle represent the lipid number in the enriched categories $(P<0.05)$. 


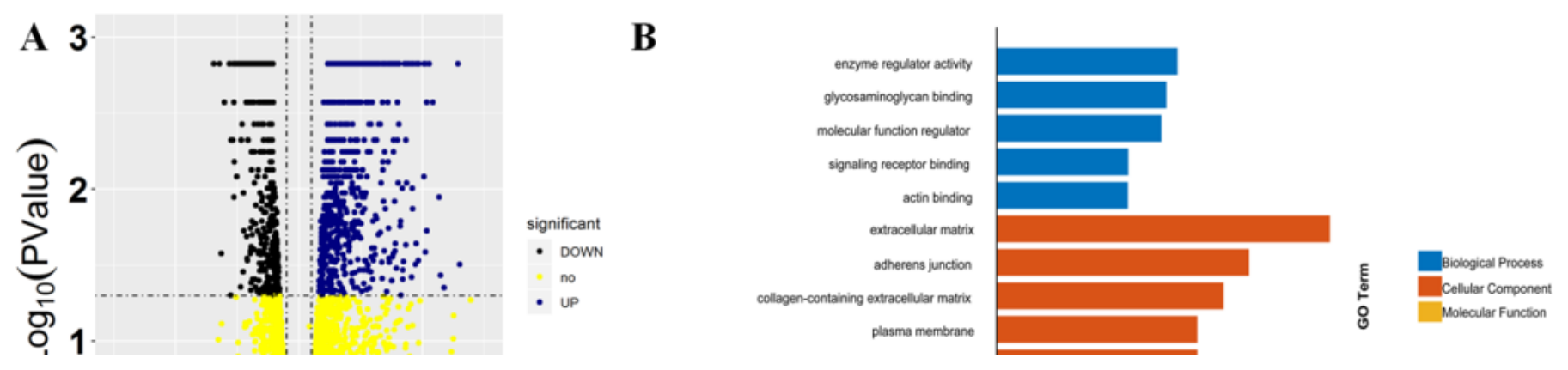

Figure 5

Differentially expressed genes and functional enrichment analysis of BAM infected by MTB and MB. A, C: Volcanic map of gene expression in BAM after MB (A) and MTB (B) infections (Log1.5FC <-1.0 or > 1.0; qvalue $<0.05)$. B, D: All differentially expressed genes infected by $M B(B)$ and MTB (D) were divided into three $\mathrm{GO}$ categories (i.e., cell composition, molecular function, and biological process). 


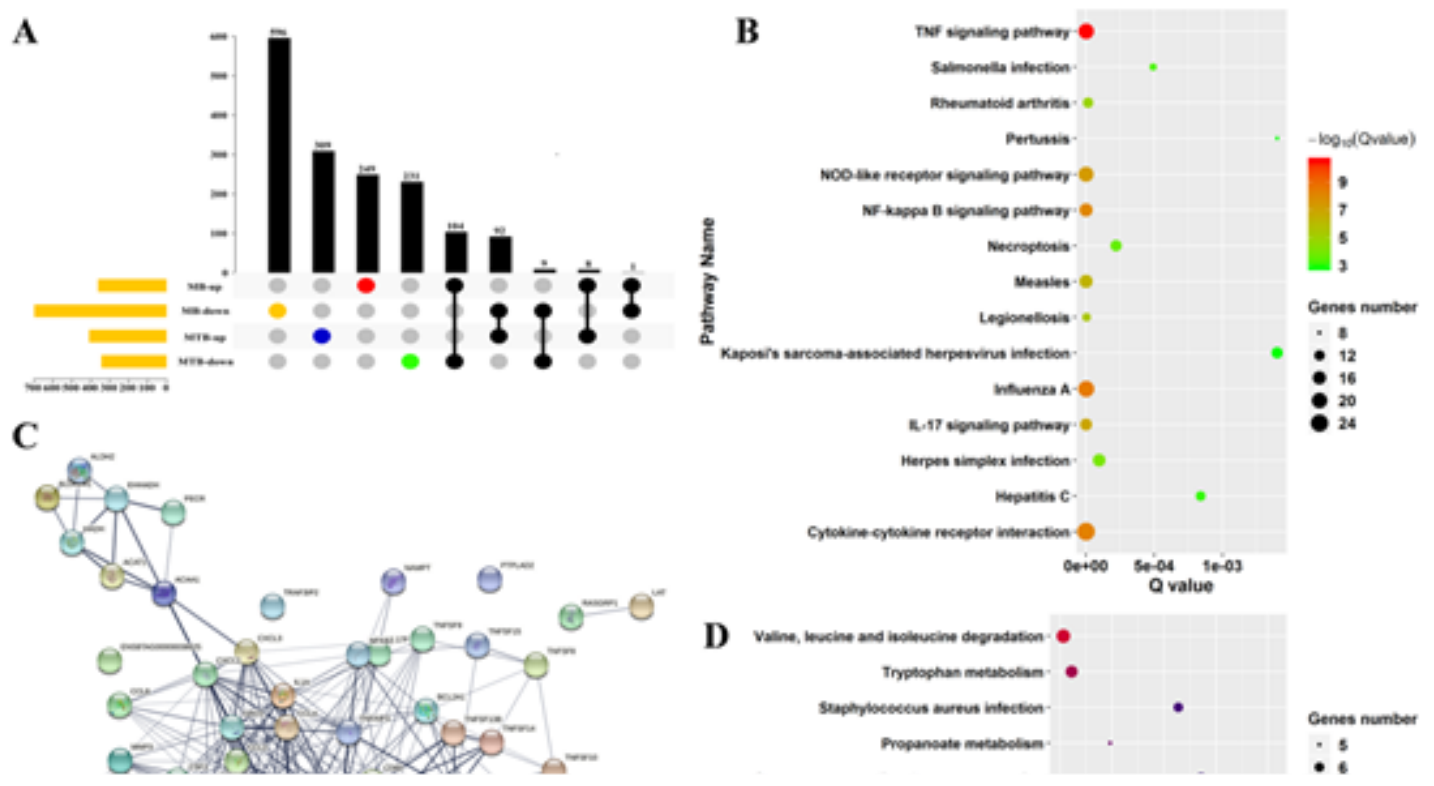

\section{Figure 6}

\section{Co-expression network and pathway enrichment analysis of key genes involved in the BAM lipid}

metabolism. A: Up-set diagrams representing the overlap of identified differentially expressed genes in both MTB- and MB-infected groups. B: Enrichment results of differentially expressed genes associated with MTB-infected BAM. The color shows the significance of pathways. The number of genes involved in the pathway is represented by the dot size. C: Protein-protein interaction network between genes involved in lipid metabolism and signaling pathways. Each circle represented an individual protein. D: Pathway enrichment result of genes associated with MB infection. 